\title{
Planning with Abstract Learned Models While Learning Transferable Subtasks
}

\author{
John Winder, ${ }^{1}$ Stephanie Milani, ${ }^{2}$ Matthew Landen, ${ }^{3}$ Erebus Oh, ${ }^{1}$ \\ Shane Parr, ${ }^{4}$ Shawn Squire, ${ }^{1}$ Marie desJardins, ${ }^{5}$ and Cynthia Matuszek ${ }^{1}$ \\ ${ }^{1}$ University of Maryland, Baltimore County, ${ }^{2}$ Carnegie Mellon University, ${ }^{3}$ Georgia Institute of Technology, \\ ${ }^{4}$ University of Massachusetts Amherst, ${ }^{5}$ Simmons University
}

\begin{abstract}
We introduce an algorithm for model-based hierarchical reinforcement learning to acquire self-contained transition and reward models suitable for probabilistic planning at multiple levels of abstraction. We call this framework Planning with Abstract Learned Models (PALM). By representing subtasks symbolically using a new formal structure, the lifted abstract Markov decision process (L-AMDP), PALM learns models that are independent and modular. Through our experiments, we show how PALM integrates planning and execution, facilitating a rapid and efficient learning of abstract, hierarchical models. We also demonstrate the increased potential for learned models to be transferred to new and related tasks.
\end{abstract}

\section{Introduction}

Model-based reinforcement learning (RL) acquires a model of an agent's interaction with its surroundings. In this set of approaches, the learned model captures the stochastic effects of actions on future states and rewards. Once a model has been acquired, an agent can use it for probabilistic planning to anticipate behaviors that will lead to a well-chosen path through state space.

For challenging tasks in a complex environment, one common technique is to "divide and conquer," breaking down an overall task into a hierarchy of separate, smaller subtasks that are easier to solve and, ideally, reusable. Such subtasks are commonly represented and variously discussed in RL literature as skills (Konidaris, Kaelbling, and LozanoPerez 2018) and options (Sutton, Precup, and Singh 1999).

However, in practice, hierarchical reinforcement learning (HRL) often relies on an expert's manual encoding of domain knowledge to design hierarchies of subtasks. These methods directly encode a designer's preconceptions and bias, due to the specification of subtasks' structure and scope. For example, HRL techniques such as MAXQ (Dietterich 2000), HAM (Parr and Russell 1998; Bai and Russell 2017) and AMDP hierarchies (Gopalan et al. 2017), as well as most work on options, depend on experts specifying an interlocking hierarchy of subtasks. Methods that do learn structure, such as MLSH (Frans et al. 2017) and HIRO (Nachum et al. 2018), still predetermine properties

Copyright (c) 2020, Association for the Advancement of Artificial Intelligence (www.aaai.org). All rights reserved. like the depth and width of the hierarchy. While option/skill discovery research attempts to address these limitations, many assumptions currently remain, such as ad hoc heuristics for transfer mappings, how state abstraction functions are acquired (MacGlashan et al. 2015). As a result, it remains a significant issue that learned subtasks are specific to the context in which they are learned, both the training environment and the hierarchy in which they are embedded.

The ultimate aim of this research is to develop a method by which an agent can autonomously construct a robust hierarchy of subtasks and their models. To support planning and task transfer, these subtasks should: (1) be independent of one another, meaning that each subtask is a complete Markov decision process unto itself, solvable without relying on other subtasks; and (2), be modular, meaning that they can be swapped in or out of a hierarchy. Intuitively, these constraints encode the requirement that subtasks can be added and removed without breaking the learned hierarchy and can be transferred to new tasks.

We introduce Planning with Abstract Learned Models (PALM), a novel method for assembling task components into subtasks with self-contained, local models. When deployed to a new task, PALM computes each subtask's model while preserving its independence. The main contributions of this work are as follows:

1. Lifted Abstract Markov Decision Process (L-AMDP): an independent, modular subtask representation.

2. PALM Phase-2: a process for converting task hierarchies learned from demonstrations into lifted AMDPs.

3. PALM PhaSe-3: an algorithm for acquiring transition models for all useful subtasks in a hierarchy via integrated planning and learning.

\section{Background \& Related Work}

Our work on learning hierarchies of independent, modular subtasks is in the general research area of reinforcement learning (RL). An RL scenario considers an agent that executes actions determined by some policy, receives feedback (reward), observes the change in its world state, and updates its policy to increase the expected reward.

Such scenarios are defined by Markov decision processes (MDPs), $M=\langle\mathcal{S}, \mathcal{A}, T, R, \gamma\rangle$, comprised of: a set of states $\mathcal{S}$; a set of actions $\mathcal{A}$; a transition probability distribution $T$ : 


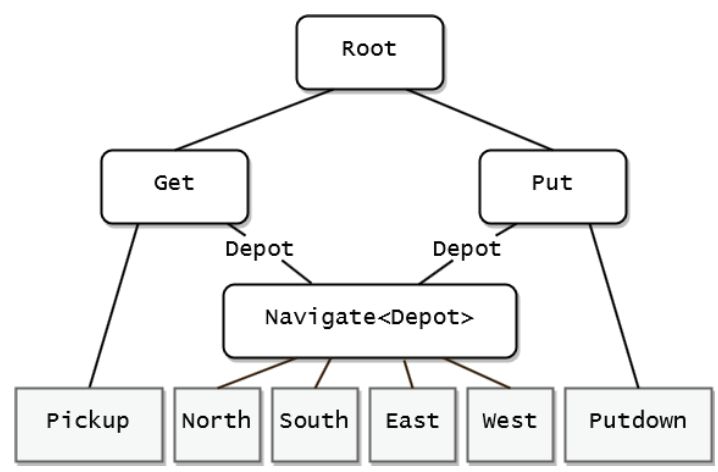

(a) PALM-ET.

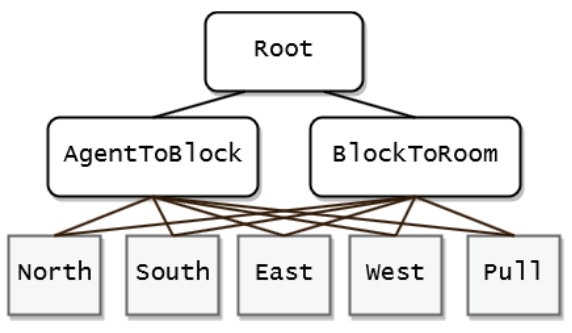

(b) PALM-EC.

Figure 1: PALM-ET uses the classic expert Taxi hierarchy from Dietterich (2000), parameterized for multiple passengers. PALM-EC is our expert hierarchy for Cleanup.

$\mathcal{S} \times \mathcal{A} \times \mathcal{S} \rightarrow[0,1]$; a reward function $R: \mathcal{S} \times \mathcal{A} \times \mathcal{S} \rightarrow \mathbb{R}$; and a scalar discount factor $\gamma \in(0,1]$, which governs the importance of future rewards. The agent's goal is to find the most rewarding behavior over time, as represented by a probabilistic policy $\pi: \mathcal{S} \times \mathcal{A} \rightarrow[0,1]$. That policy is often learned through the computation of a value function $V^{\pi}$, which captures the long-term value of being in a given state. Value is computed recursively over rewards received from following the policy through state-action space as $V^{\pi}(s)=\mathbb{E}_{\pi}\left[\sum_{k=0}^{\infty} \gamma^{k} r_{t+k+1} \mid s=s_{t}\right], \forall s \in \mathcal{S}$. The optimal policy $\pi^{*}$ maximizes cumulative expected future rewards, corresponding directly to an optimal value function.

Hierarchical RL (HRL) is commonly used in situations that require agents to perform repeated sequences of actions over an extended period. HRL decomposes a hard task into subtasks that are more focused, more manageable, and (ideally) repeatable. Two notable HRL techniques are options (Sutton, Precup, and Singh 1999) and MAXQ (Dietterich 2000). Both accomplish temporal abstraction, in which the agent reasons over actions that unfold over many time-steps rather than discrete, atomic actions. HRL is also conducive to state abstraction, in which similar grounded states are aggregated (Boutilier and Dearden 1994; Abel et al. 2018). Combined, these make HRL well suited to address many open challenges in RL, namely mitigating the curse of dimensionality by reducing the state-action space, facilitating generalization across similar (abstract) states, and leveraging transfer learning across tasks.

Central to HRL is the notion of a task hierarchy, a graph- ical structure that encodes how subtasks and primitive actions relate (for example, what subtasks use other subtasks or actions). Thus, each subtask may be viewed as a node in a directed acyclic graph (the hierarchy). MAXQ, for example, decomposes an MDP into a set of smaller MDPs - one per subtask - with transitions and rewards derived from the base MDP. It then computes the overall value function recursively down through each possible branch, using a piece-wise completion function to determine the expected discounted reward contributed by each subtask.

A drawback in much HRL research is the reliance on humans to design the task hierarchy. As a result, there has been significant research on autonomously learning such hierarchies. Prior efforts in learning hierarchical structures of tasks include discovering sub-MDPs divided by suitable boundary "exit" states (Hengst 2002; 2004); acquiring transferable option policies directly (Brunskill and Li 2014; Topin et al. 2015); learning high-level skills and abstract actions from demonstrations (Konidaris, Kaelbling, and LozanoPerez 2018); learning hierarchies based on associating actions with relevant state features via CSRL (Li, Narayan, and Leong 2017); and, causally-annotated approaches that produce MAXQ-style task graphs, such as VISA (Jonsson and Barto 2005; Vigorito and Barto 2010), HI-MAT (Mehta et al. 2008), and its extension, HierGen (Mehta 2011).

While we restrict our examination in this paper to traditional, symbolic RL, there is a rich and growing body of work in the context of deep HRL (Kulkarni et al. 2016). MLSH (Frans et al. 2017) learns master- and sub-policies jointly, with the former acting as a controller over the latter group. The HIRO agent (Nachum et al. 2018) creates two tiers of policies, in which the lower level interacts with the grounded MDP based on goals directed by the higher level. Notably, HIRO eschews any goal representation, so it lacks an explicit notion of subtask. Along this thread, a HAC agent (Levy et al. 2019) does specifically consider goals and subtasks. HAC combines hindsight experience replay with universal function approximators in an algorithm that learns, in parallel, multiple levels of policies. Finally, the unified model-free HRL algorithm (Rafati and Noelle 2019) combines intrinsic motivation with aspects of both skill and subgoal discovery.

One drawback of existing hierarchical methods, such as planning with (predefined) AMDP hierarchies (Gopalan et al. 2017), is the requirement of human intervention or curation at some (or many) parts of the design. Examples include defining how subtasks chain together, picking the number or depth of subtasks, providing the low-level controllers beforehand. This work is motivated by the goal of learning subtasks, both their relations among each other and their internal model dynamics, without expert knowledge. While several HRL techniques can suffer from nonstationarity issues arising due to learning multiple levels of subtasks (Nachum et al. 2018), our technique is devised to counter the problem without an impact to performance. Lastly, in our approach, PALM learns AMDP subtasks that are independent and modular. As such, these AMDPs can be removed or added without impairing the functioning of other subtasks or the hierarchy as a whole. Taken together, the method we 
introduce facilitates the inclusion of new skills on transfer to different and more difficult tasks.

We draw a connection between PALM and an existing approach in planning and RL focused on abstract modular and independent tasks. PLANQ-learning (Grounds and Kudenko 2005) synthesizes a hierarchical variant of Qlearning with STRIPS planning (Fikes and Nilsson 1971) to reason further into the future over a sequence of subtasks. PPSB (Segovia-Aguas, Ferrer-Mestres, and Jonsson 2016), planning with partially specified behaviors, builds on PLANQ with formalisms to afford more modular subtask decomposition. PLANQ and PPSB encode preconditions and rules that comprise a knowledge base to inform per-subtask policies that are then learned independently, as guided by high-level plans. PPSB is similar to our approach, though instead of AMDPs, PPSB relies on the partial specifications of abstract actions based in STRIPS. This framing requires expert-created conditions and operators for actions, including the models of the abstract actions. PALM and LAMDPs do not encode such information beforehand, except incidentally through state abstraction, which we show can itself be learned from data without human supervision, due to the generality of AMDPs. PPSB also assumes no dead-ends and determinism within subtasks. In contrast, PALM's recursive algorithm is designed to be tolerant to stochasticity and accounts for subtask failures explicitly. On encountering a failed subtask, PALM escapes out and recurses up to replan at the appropriate parent AMDP.

\section{Approach}

PALM aims to remedy issues of existing techniques in a three-phase framework. In PHASE-1, our approach first relies on input sample demonstrations and a hierarchy learner, producing a MAXQ-like hierarchy. Our contributions begin in PHASE-2 with a procedure for creating a new subtask representation, the L-AMDP, from those extracted in PHASE1. PHASE-3 then describes our novel algorithm for learning those subtask models in a hierarchy simultaneously at multiple levels, while preserving their self-contained properties.

\subsection{Learning from Demonstrations}

PHASE-1 uses existing HRL techniques to learn a MAXQstyle task hierarchy from demonstrations. MAXQ decomposes a task hierarchically across subtasks, computing the value function by defining completion function to represent the amount of expected discounted reward recursively credited down through each branch of a task's subtasks. Hierarchy-learning algorithms are typically assumed to have access to sample trajectories of an agent exploring and solving the domain (e.g., state-action-reward transition tuples), labeled with, minimally, whether the sample is a success or failure.

Given this set of solution demonstrations, $\mathcal{D}$, PHASE1 takes any general existing algorithm $\mathrm{H}$ that assembles a MAXQ-style task graph, $\mathrm{H}(\mathcal{D}) \rightarrow G$. This phase assumes the use of factored state space MDPs, such as states that are defined simply in terms of a feature vector. There exist many possibilities for $\mathrm{H}$ (Mehta 2011; Li, Narayan, and Leong
2017; Levy et al. 2019); we use HierGen (Mehta 2011) because it creates a per-subtask state abstraction mapping. Alternatively, PHASE-1 can be skipped if an expert supplies the task graph $G$ (a hierarchy of MAXQ subtasks).

\subsection{Assembling Lifted Abstract MDP Subtasks}

Given an expert or learned task graph from the previous phase, we present a novel process to convert all of its subtasks into a more general abstract MDP representation, PHASE-2 of the process. We refer to this novel intermediate representation of an abstract subtask as the Lifted AMDP, or L-AMDP. At a high level, PHASE-2 maps the task graph $G$ to $H$, a hierarchy of Lifted AMDPs.

We depart from standard approaches by representing subtasks abstractly as decision problems complete unto themselves. To do so, we redefine subtasks using the formalization of abstract MDPs (AMDPs) (Gopalan et al. 2017). AMDPs differ from the more familiar framing of subtasks as skills or options in that each AMDP subtask has its own local model of reward and transitions, from which value functions and policies may be generated.

Formally, an AMDP subtask is an MDP with abstract components, $\tilde{M}=\langle\tilde{\mathcal{S}}, \tilde{\mathcal{A}}, \tilde{T}, \tilde{R}, \gamma, \tilde{\mathcal{G}}, \tilde{\mathcal{F}}, \phi\rangle$, consisting of: abstract actions $\tilde{\mathcal{A}}$, comprised of child subtasks (e.g., other AMDPs or primitive actions from $M$ ); sets of terminal goal and failure states $\tilde{\mathcal{G}}, \tilde{\mathcal{F}} \subset \tilde{\mathcal{S}}$; and (optionally) state abstraction $\phi: \mathcal{S} \rightarrow \tilde{\mathcal{S}}$, which maps ground states to abstract state space by aggregating similar states or removing features.

Extending this concept, lifted AMDPs are parameterized over features (state factors). An L-AMDP contains a goal predicate $\tau$, a failure predicate $\chi$, subtask parameters $\theta$ (if any), and a state abstraction function $\phi$. We formally define an L-AMDP as $\tilde{M}_{\theta}=\langle\cdot, \tilde{\mathcal{A}}, \cdot, \tilde{R} \sim\{\tau, \chi\}, \cdot \tilde{\mathcal{G}} \sim\{\tau\}, \tilde{\mathcal{F}} \sim$ $\{\chi\}, \phi\rangle$. On grounding to a target task MDP, they serve as AMDP subtasks with models missing; the remaining components are induced or learned during PHASE-3.

PHASE- 2 is a procedure that builds a hierarchy of Lifted AMDPs, $H$, from a task graph $G$. Construction of L-AMDPs occurs one at a time, following a reverse topological ordering of $G$. Intuitively, PHASE-2 first wraps primitive actions (leaf nodes of $G$ ) into "subtasks," then creates one L-AMDP subtask for each composite action (internal node of $G$ ), and ends by making a subtask for the root. There is always a root subtask, with terminal conditions identical to the task MDP.

Given our assumption of factored states, each subtask either already possesses terminal conditions. Or, we can train a classifier to learn goal and failure predicates based on terminal states in $\mathcal{D} . \tau$ and $\chi$ differ from the termination predicate in MAXQ by capturing success and failure separately. This property of L-AMDPs is crucial for $\tilde{R}$, the induced pseudoreward function. We make the standard assumption that subtasks adhere to a goal-fail structure. In this scheme, the highest reward is received at a goal state, the most negative reward is received at any failure state, and a default reward is otherwise observed; this assumption simplifies and bounds the shape of $\tilde{R}$. Although $\tilde{R}$ has a known shape, a conditionbased pseudo-reward may be included as well, either learned from $\mathcal{D}$, or added manually. Thus $\tilde{R}$, like $\tilde{T}$, once grounded, 
must be learned to a task MDP in the next phase, during execution.

We use the (optional) $\theta$ list of parameterizable features. A parameterized L-AMDP may generate multiple realized AMDPs, one for each possible grounding in the target task. For example, the Taxi hierarchy's NAVIGATE subtask (Figure 1a) may be grounded once per destination depot in a given MDP. HierGen (Mehta 2011) provides a straightforward way of generating $\theta$ based on what factors are checked or change in the trajectories $(\mathcal{D})$ for that subtask (e.g., the positional coordinates of depots in Taxi). Similarly, the state abstraction may be hand-engineered or learned, such that $\phi$ includes all features from $\tau, \chi$, and $\theta$. Finally, PHASE2 makes $\tilde{\mathcal{A}}$ by linking to all AMDPs that correspond to child subtasks of this subtask as defined in $G$; reverse topological ordering ensures these AMDPs already exist. The missing $\gamma$ will likewise be inherited from the ground MDP.

In summary, the Lifted AMDP is a novel, modular, independent subtask representation. In PHASE-2, each input MAXQ task node is converted into an L-AMDP.

Subtask Independence. The L-AMDP subtask representation devised for PALM leads to independently computable models. In possessing each component of an MDP, an AMDP is itself an MDP, and can be solved using any standard approach. Deployed to some task (PHASE-3), whenever the agent first executes an L-AMDP subtask, it is automatically grounded to its particular circumstance, with the missing pieces induced or learned gradually through experience. Computing plans inside a grounded AMDP subtask is therefore self-contained: it may be solved as with any MDP.

This is an improvement over dependencies present in both options and MAXQ subtasks. Computing the hierarchical value function for a MAXQ subtask requires decomposing via the completion function into its child subtasks. While this in principle reduces the size of the value function, it acquires this property by entangling subtasks with ones below it. R-MAXQ (Jong and Stone 2008), the model-based extension of MAXQ, highlights the complexity of learning MAXQ subtask models: its recursive variant of the Bellman equation must consider the stochastic transitions across all child subtasks, for all their possible terminal states. For options, approximating an option's multi-time model requires knowing all of the (possibly infinite) number of time-steps across its subtasks (Abel et al. 2019). In general, computing option plans in complex hierarchies requires solving how their models compose jointly, in a dependence akin to that of the MAXQ completion function (Silver and Ciosek 2012). The practical effect of L-AMDP subtask independence is that, on grounding, subtasks may be computed directly, in isolation, without any recursive decomposition.

Subtask Modularity. L-AMDPs also improve the modularity of subtasks: unnecessary subtasks can easily be pruned, and new ones incorporated. For example, imagine transferring from the classic Taxi task to a new task that requires refueling. The existing hierarchy (Figure 1a), defined in terms of L-AMDPs, can be transferred directly; the

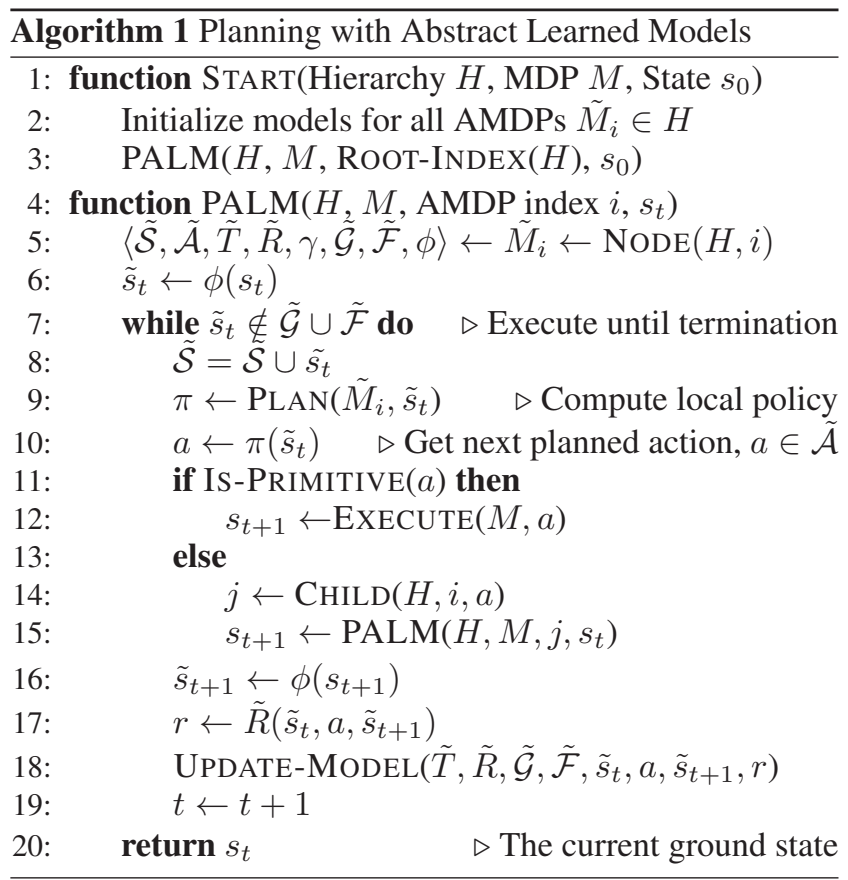

learned models of its abstract subtasks are still valid, due to the AMDP's state abstraction and independent model. All that needs to be added is a newly initialized REFUEL subtask, included as a child of the ROOT subtask, with its set of abstract actions $\tilde{\mathcal{A}}$ containing NAVIGATE and a primitive action for refueling (assuming it is available in the task MDP).

When a new subtask is added to a hierarchy, its presence would be included in any parent subtasks' abstract action set; only the new subtask and any parents need to be updated. Assuming an optimism-under-uncertainty modelbased scheme is used, the newly available transitions due to the new subtask are incentivized to be explored. Removing a subtask is likewise straightforward: the subtask only needs to be removed from its parents' $\tilde{\mathcal{A}}$. Any learned transitions involving that action would, thus, be disallowed or deleted.

\subsection{Integrating Planning and Learning}

PHASE-3 sees a new model-based hierarchical RL algorithm (Algorithm 1) applying the hierarchy from PHASE-2 to a new (previously unseen) task MDP $M$. This algorithm recursively integrates planning and learning to acquire its subtasks' models while solving $M$. We refer to the algorithm as PALM: Planning with Abstract Learned Models.

In model-based RL, the agent uses its observations to bootstrap an approximation of the model, $\tilde{T}$ and $\tilde{R}$, which express the probabilistic effect of actions on the environment. In our case, this occurs at multiple levels of abstraction, with PALM learning the $\tilde{T}$ and $\tilde{R}$ of each subtask, updating the model after using it to make a decision. Conceptually, PALM plans the solution to a given task (a policy $\pi$ ), selects a subtask, recurses, and continues this process successively until reaching and executing a primitive action of $M$. Thus, planning and execution are interleaved.

The inputs of PALM are $M$, an initial ground state $s_{0} \in$ 
$\mathcal{S}$, and a hierarchy $H$ of L-AMDPs. Planning begins at the root subtask. With each recursive call to PALM, $s_{t}$ is projected into the given AMDP's state space by applying state abstraction. PALM then computes a local policy $\pi$ for the AMDP, selecting the next planned action. If that action is primitive $(a \in \mathcal{A}$ ), executing it in the base environment causes a "real" transition in the world, and it returns the next ground state, $s_{t+1}$. Otherwise, PALM retrieves the linked child subtask in the hierarchy, and recurses down to it, repeating this process. On completion of the execution step or recursive call, PALM abstracts the new ground state, $\tilde{s}_{t+1}$, obtains the pseudo-reward $r$ for the abstract transition, and updates the current AMDP's model, recomputing the approximation of $\tilde{T}$ and $\tilde{R}$ based on the observed transition.

It is desirable for PALM to be a general framework. PHASE-3 is designed to permit the use of any MDP planner, such as Value Iteration, UCT (Kocsis and Szepesvári 2006) methods like PROST (Keller and Eyerich 2012), or bounded RTDP (McMahan, Likhachev, and Gordon 2005). PALM also supports any standard model-based approach such as $E^{3}$ (Kearns and Singh 2002) or R-MAX (Brafman and Tennenholtz 2002). Additionally, because subtasks are independent, they may use different planners as appropriate-for example, if value function approximation is required in one subtask but not another.

Avoiding Nonstationarity. One common issue in HRL is that of nonstationarity: so long as a lower-level model has not converged to a stable point, it defines a moving target for any higher-level subtask relying on that model. Learning on a nonstationary process upends the assumptions needed in RL to update via bootstrapping and guarantee optimality. To avoid the effects of nonstationarity, we rely on the "knows-what-it-knows" (KWIK) framework. A KWIK algorithm such as R-MAX (Li et al. 2011; Szita and Szepesvári 2011) reasons explicitly about known or unknown transitions. We employ a strategy of solidifying lower subtask models first, then learning successively higher levels. During execution, PALM simply returns a signal indicating the known/unknown status of the transition that just occurred, informing the parent task if it should ignore or process its own transition. In effect, subtask models are cemented before their parent models are learned. Without this update strategy, we find empirically that PALM produces solutions swiftly while learning on nonstationary models, often achieving more optimal behavior; however, this strategy ensures optimality across levels of abstraction such that PALM converges to a recursively optimal policy.

Complexity. We consider the complexity of each AMDP subtask individually. In the execution of PALM on a given AMDP (line 9), computational complexity is dominated by the planning algorithm. In the worst case, this planner is recomputed at each step. The sample complexity for an AMDP subtask in general will be $O(\rho)$, where $\rho$ is the sample complexity of the model-learning algorithm used; for RMAX, which we use, $\rho=|\mathcal{S}|^{2}|\mathcal{A}| V_{\max }^{3} \epsilon^{-3}(1-\gamma)^{-3}$ given $M$ 's maximum value $V_{\max }$ and the PAC-MDP parameter $\epsilon$

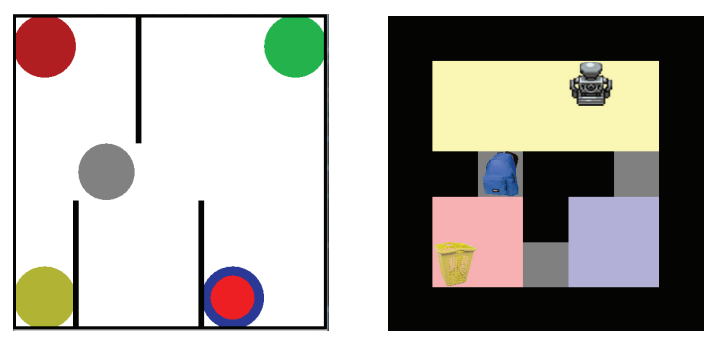

Figure 2: Example starting states pulled from a distribution over possible tasks. We use the factored OO-MDP representation for tasks (Diuk, Cohen, and Littman 2008). A Taxi task, left, contains the agent "taxi" (gray), walls, depots, and passenger (red). A Cleanup task, right, includes blocks, doors, rooms, and the agent "robot" facing north.

(Li 2012). R-MAX is a well-studied, PAC-MDP algorithm, with guarantees of convergence and bounded space and sample complexity (Strehl, Li, and Littman 2009). Thus, hierarchies of fewer nodes and shallower depth are preferable in terms of sample complexity.

\section{Experimental Methodology}

We compare our method against R-MAXQ (Jong and Stone 2008), the most closely aligned existing algorithm to PALM and its aims. R-MAXQ unites R-MAX with MAXQ, achieving a model-based approach of the latter method's value function decomposition technique by specifying a recursive variant of the Bellman operator. As opposed to other HRL algorithms, R-MAXQ is similar to PALM in representing abstract models concretely; unlike PALM, R-MAXQ's models do not meet the criteria we desire for subtask independence and modularity. To keep our PALM in line with RMAXQ, we parallel it by using Value Iteration as the planner and R-MAX as the model-based RL algorithm for all subtasks; these are baselines, and due to subtask independence, more sophisticated techniques could be used as appropriate.

Domains. The Taxi domain (Dietterich 2000) is a common HRL problem where the agent, a taxi, must collect passengers and ferry them to different destinations. The Cleanup domain simulates a robot that tidies a house by putting blocks where they belong, similar to the game of Sokoban (MacGlashan et al. 2015; Guez et al. 2019). The agent must navigate a grid-world composed of rooms and blocks, pushing and pulling blocks until one (or more) target blocks are in a room of a matching color. Cleanup tasks appear deceptively simple. They present a combinatorial explosion of state space as more blocks and rooms are added. Moreover, agents frequently encounter edge cases and bottlenecks that are difficult to exit (e.g., blocks in a corner). Visualizations of a classic Taxi task and a Cleanup task are shown in Figure 2.

Hierarchies. All PALM methods use a hierarchy of LAMDP subtasks that, on each trial, are grounded to a new, random target MDP. The lifted hierarchies we describe use 


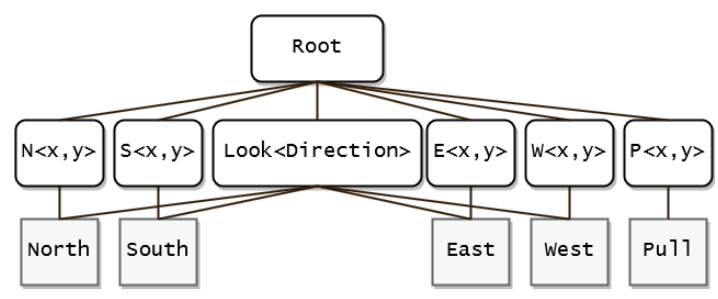

Figure 3: PALM-AC, amended by experts.

an abbreviation scheme: $\mathbf{E}$ is an expert-designed hierarchy, $\mathbf{H}$ is a HierGen-learned hierarchy, $\mathbf{A}$ is an amended hierarchy that updates a learned hierarchy with expert knowledge, $\mathbf{T}$ is a hierarchy for Taxi, and $\mathbf{C}$ is a hierarchy for Cleanup. Thus, PALM-ET and PALM-HT are the expert and learned hierarchies for the Taxi domain, following directly from Dietterich (2000) and Mehta (2011), respectively. PALM-EC (Figure 1b), our expert hierarchy for Cleanup, decomposes the task in terms of moving to a block and then the block to a room. PALM-HC, the learned Cleanup hierarchy, degenerates into a flat hierarchy without subtasks beyond ROOT, which contains only the primitive actions. We find this negative result (from our use of HierGen) surprising, and discuss it further later. Informed by HC, PALM-AC (Figure 3), is an amended, second-draft expert hierarchy for Cleanup. In $\mathrm{AC}$, each primitive action is wrapped in an AMDP subtask parameterized by the $\mathrm{x}-\mathrm{y}$ coordinates of the destination relative to the agent position. Additionally, we define a LOOK task for turning to look in a direction (without hitting a wall), which promotes an agent's ability to plan to face a block before pulling it. Combining these parameterized, shielded primitive actions with state abstraction means AC ignores all irrelevant features, and never plans an action that is illegal or results in a self-transition.

\section{Experimental Results}

We examine the usefulness of learned hierarchies in comparison with expert-specified hierarchies, and we consider the performance of PALM in terms of the cumulative steps taken and reward acquired across episodes. Our figures are shown with the $95 \%$ confidence region shaded.

\subsection{Baseline Results}

Figure 4 compares PALM with R-MAXQ. In a small Taxi task $(1 \times 5$ grid $)$ with deterministic transitions, R-MAXQ exceeds PALM when using the same hierarchical structure (ET). In Figure 4b, we observe a different asymptotic relationship when facing stochasticity. R-MAXQ struggles to compute the recursive models of its subtasks, even in this task with only 100 states. Note that these results match those reported in Jong and Stone (2008), and belie the fact that RMAXQ does ultimately approximate correct models, just in episodes beyond those shown.

These results are even more stark in Cleanup, due to the exacerbation of R-MAXQ's scaling issues in handling larger, more complex state spaces. R-MAXQ experimentally requires orders of magnitude more computation time than

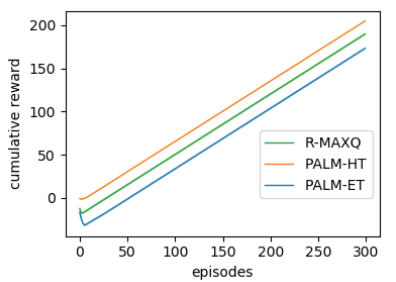

(a) Small, deterministic Taxi.

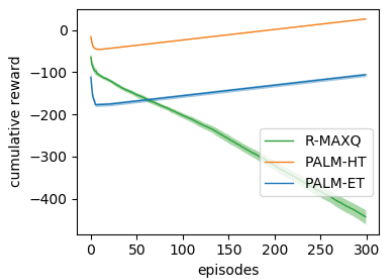

(b) Classic fickle Taxi.
Figure 4: In 4a, without randomness, all approaches rapidly find an optimal policy (reflected in their asymptotic trends). In 4b, ET and HT again converge quickly due to the ease of computing AMDP models relative to R-MAXQ's approach. HT consistently learns with fewer samples; while HT also learns fewer models, they are larger and more complex.

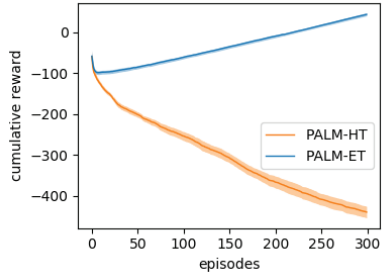

(a) Classic, 2 fickle passengers.

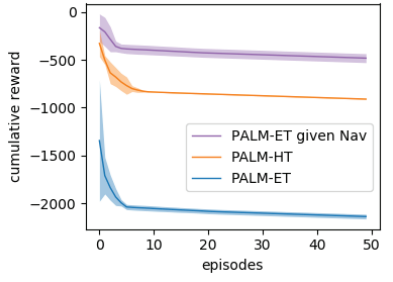

(b) Large, 1 passenger.
Figure 5: In 5a, multiple fickle passengers cause HT to vacillate while learning mid-level models. In $5 \mathrm{~b}$, we provide a converged model of the navigation subtask to an ET agent, highlighting the benefit of transferring learned models.

PALM for the same domain. To highlight the stark difference in the actual time operations between the two methods, we report the empirical runtime difference of 20 trials on a small Cleanup task with $|\mathcal{S}|$ in the hundreds. Using EC for both, PALM decreases from $100 \mathrm{~ms}$ per episode to 10 once its models have converged, while R-MAXQ consistently averages $10^{4} \mathrm{~ms}^{1}{ }^{1}$ Behaviorally, PALM quickly converges towards a near-optimal policy while R-MAXQ conducts excessive exploration. We observe that R-MAXQ continually expands down branches of a hierarchy that are unhelpful to solving the overall goal, for example, continually re-entering a room and going to as many spaces as possible and facing in all directions. This difference is due to subtask independence: unlike with MAXQ, planning with an AMDP hierarchy expands only those child subtasks actually used in the rollout of a hierarchical policy (Gopalan et al. 2017). Thus, PALM's focused expansion learns models that solve the harder Cleanup tasks quickly, where R-MAXQ has superfluous branching.

\subsection{Results for Expert vs. Learned Hierarchies}

We now consider the impact of learning a hierarchy. Figure $4 \mathrm{~b}$ shows how HT, a hierarchy learned from data without human supervision, can surpass the efficiency of one made

\footnotetext{
${ }^{1}$ Performed on i7-4790K CPU @ 4.00 GHz, 20GB of RAM.
} 

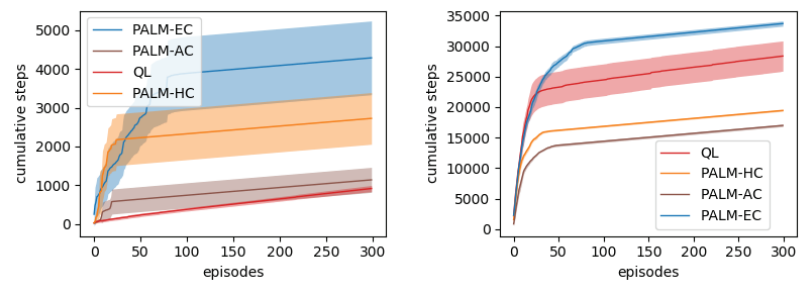

(a) 1 block, 3 rooms, $5 \times 7$ grid.

(b) 1 block, 3 rooms, $7 \times 7$ grid.
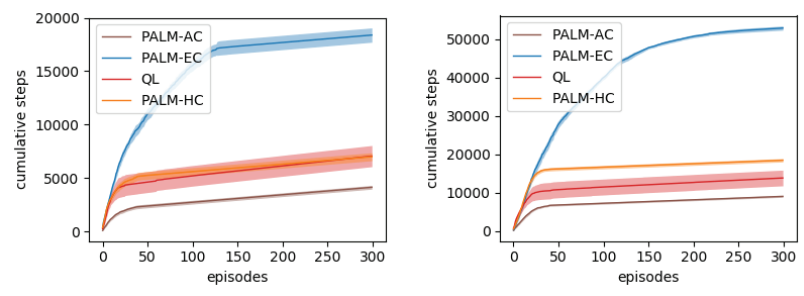

(c) 2 blocks (1 target), $5 \times 5$ grid.

(d) 2 blocks, $5 \times 5$ grid.

Figure 6: Cumulative steps for the Cleanup domain, highlighting the number of samples needed to reach convergence.

by an expert, ET, with the former attaining roughly the same asymptotic performance as ET in only a fraction of the samples required. PALM makes it possible to create everything needed for hierarchical planning via sampled experiences without expert knowledge or intervention.

In Figure 5, we examine hierarchies on increasingly more complex tasks. However, ET achieves greater cumulative reward in the successive experiments than HT, with the latter faltering to increasingly greater extents as more passengers are added. HT reasons about putting down passengers in its top-most AMDP, slowing it down when there are more than one, whereas ET more cleanly separates the process of retrieving and depositing passengers. As we scaled to variants with three and four passengers, we found this trend continued to worsen for HT, while ET could achieve greater cumulative reward in the successive experiments, scaling more gracefully in contrast to the sinking asymptotic trend of HT. For Figure 5 b, the large Taxi variant, on a $20 \times 20$ grid with only one passenger, HT has the same lead over ET as in $4 \mathrm{~b}$.

For Cleanup, in Figure 6, we report results in cumulative steps rather than reward (each complete episode yields a reward of exactly 1.0) as complexity is varied among tasks. Specifically, we consider when there are more rooms, Figures $6 \mathrm{a}$ and $6 \mathrm{~b}$, or fewer rooms but more blocks, Figures $6 \mathrm{c}$ and $6 \mathrm{~d}$. The key property to observe in Figure 6 is that the time-to-convergence signifies how effectively the algorithm computes its models, with the "elbow" of each trend indicating the point at which the models solidify. Thus, we report cumulative instead of average steps; with the latter, it is harder to distinguish the differences in the long-term modellearning trends among the algorithms (typically, they all eventually have the same average number of steps once their models have converged). We note that the asymptotic rela- tions among the PALM methods hold across all tasks: EC requires the most samples, while $\mathrm{HC}$ is in the middle, and the redesigned $\mathrm{AC}$ solves the domains most readily. Both $\mathrm{HC}$ and $\mathrm{AC}$ outperform QL, which we include to benchmark model-free learning (without planning). In comparison, the original expert design, EC, is much less effective, taking statistically significantly more steps to learn than QL, despite having fewer models than AC. AC is created based on our observations of EC and then $\mathrm{HC}$; the fact this second iteration expert hierarchy performs better after observing a learned one highlights the value of human cooperation with learning algorithms in designing solutions.

We note a finding that emphasizes the challenge of generalization that endures for hierarchy-learning algorithms. Specific to the algorithm we used, HierGen, we observed it would degenerate if trained on $\mathcal{D}$ of sufficient environmental complexity. For example, if $\mathcal{D}$ contained two Cleanup MDPs with the same number of rooms and blocks but no shared features (e.g., colors or door locations), HierGen would produce only flat MAXQ graphs (causally-annotating subsequences into a precedence graph of solely one node). Despite this degeneration, we remark that the resulting $\mathrm{HC}$ still produces valid, reasonably efficient solutions in PHASE-3. In each case, it converges significantly faster than EC.

\subsection{Independence, Modularity, \& Transfer}

The most significant difference between our formulation and R-MAXQ, and other subtask representations more generally, is that PALM yields independent models. Practically, PALM circumvents the typical HRL approach of creating interdependent "puzzle piece" forms of temporal abstraction that must link together precisely. For example, options must terminate where another can initiate, skills require chaining from target to target, and MAXQ does not distinguish between goal and failure termination predicates. As a result, we identify a key benefit of PALM: graceful replanning on failure. Should a subtask fail, control simply returns to its parent, and so on as needed, to the appropriate level for replanning, and a new plan is generated.

We also highlight the transferability implications of subtask modularity: PALM can learn one subtask, then transfer its abstract models to new, related tasks, greatly accelerating overall performance. To transfer in a model, it is first necessary to obtain either an expert-defined one or a learned one acquired via training on an MDP sampled from the same universe.

We include an example of transfer in Figure 5b, and refer to standard metrics for transfer in RL (Taylor and Stone 2009). In this case, a converged model for the NAVIGATION subtask is transferred to a hierarchy deployed on a new task set. Here, only the high-level AMDP models need to be recomputed. This "PALM-ET given Nav" agent achieves, as expected, an immediate and statistically significant jumpstart over the algorithm with the same hierarchy, ET, as well as having a shorter time-to-convergence and greater total reward. Navigation dominates the sample complexity; however, having been learned once, it may be reused. Thus, transferred knowledge in PALM alleviates the burden of further exploration at that level, allowing the agent 
to advance more rapidly in learning higher-level AMDPs. PALM's style of encapsulated, modular transfer is impossible with R-MAXQ and other HRL methods because they learn representations that recurse down to, or ultimately depend on, the transition probabilities, reward function, and ground states specific to the given task MDP.

We would like to emphasize two major takeaways from these results. First, subtask independence limits the effects of other models on the one being learned. Since each model in PALM is computed independently, the effects of stochasticity are limited. PALM can, in effect, learn the value functions of these tasks in a more focused manner. In, for example, R-MAXQ, computational efficiency is negatively affected by the need to compute down to the primitive level at every decision point and model update; other HRL algorithms experience similar problems, requiring computation with a direct dependence on lower-level models. In a sense, they do not actually abstract away fine-grained temporal details. The multi-time model of options, for example, requires knowing the joint distribution of all possible time-steps of all options (Abel et al. 2019).

Second, PALM, unlike related approaches, produces a plan which can be revised. Our investigation of R-MAXQ on much larger, more complex MDPs (including the Cleanup tasks) indicates that the intertwined nature of R-MAXQ's computation exacerbates scalability issues. PALM's independent models inhibit such problems. Because each AMDP that PALM is learning has its own dedicated model, the model-based exploration that must occur is handled irrespective of child subtasks. This fact further distinguishes PALM: the L-AMDP subtask models are not a static policy (as with an option) or fragment of a value function (as with a MAXQ subtask), but an encapsulated MDP in its own right. As a result, each PALM subtask can decide independently, within its own confines, what actions to take, abstracting away details of child subtasks.

\section{Discussion \& Future Work}

We introduce Lifted AMDPs as novel, general, and useful representations of behavior. We develop PALM, which performs hierarchical reinforcement learning while eliminating the dependency on human authors: an agent can create a hierarchy where all constituent parts are learned entirely from data. PALM has the following novel traits. Deployed to some new task, it learns a transition and reward model for all subtasks in its hierarchy. This hierarchy itself, and any related state abstractions, may be learned from data. Models learned via PALM are transferable to related tasks. We demonstrate the effectiveness of this approach in supporting complex, hierarchical planning without human supervision. The properties of independence and modularity make our approach promising for transfer learning, training on a given task and deploying to related ones with reduced retraining and a jumpstart to performance.

PALM's ability to transfer warrants more extensive investigation, and we hope to examine its potential further, especially in cases that require mixed discrete and continuous state space L-AMDPs. These hierarchies could combine deep and traditional RL, such that subtasks needing perception or control are handled by the former and those requiring more abstract reasoning are addressed by the latter. Trained wholly in simulation to learn the higher-level symbolic models, models could be transferred over to a continuous, physical domain where only the lowest-level navigational subtasks need to be learned from scratch.

\section{Acknowledgments}

The material presented here is based in part upon work supported by the National Science Foundation under Grant No. IIS-1426452 and by DARPA under grants W911NF-15-10503 and D15AP00102.

\section{References}

Abel, D.; Arumugam, D.; Lehnert, L.; and Littman, M. 2018. State abstractions for lifelong reinforcement learning. In ICML, 10-19.

Abel, D.; Winder, J.; DesJardins, M.; and Littman, M. 2019. The expected-length model of options. In IJCAI, 19511958.

Bai, A., and Russell, S. 2017. Efficient reinforcement learning with hierarchies of machines by leveraging internal transitions. In IJCAI, 1418-1424.

Boutilier, C., and Dearden, R. 1994. Using abstractions for decision-theoretic planning with time constraints. In $A A A I$, 1016-1022.

Brafman, R. I., and Tennenholtz, M. 2002. R-MAX: A general polynomial time algorithm for near-optimal reinforcement learning. JMLR 3(Oct):213-231.

Brunskill, E., and Li, L. 2014. PAC-inspired option discovery in lifelong reinforcement learning. In ICML, 316-324.

Dietterich, T. G. 2000. Hierarchical reinforcement learning with the MAXQ value function decomposition. JAIR 13:227-303

Diuk, C.; Cohen, A.; and Littman, M. L. 2008. An objectoriented representation for efficient reinforcement learning. In ICML, 240-247.

Fikes, R. E., and Nilsson, N. J. 1971. STRIPS: A new approach to the application of theorem proving to problem solving. Artificial intelligence 2(3-4):189-208.

Frans, K.; Ho, J.; Chen, X.; Abbeel, P.; and Schulman, J. 2017. Meta learning shared hierarchies. In ICLR.

Gopalan, N.; desJardins, M.; Littman, M. L.; MacGlashan, J.; Squire, S.; Tellex, S.; Winder, J.; and Wong, L. L. 2017. Planning with abstract Markov decision processes. In ICAPS.

Grounds, M., and Kudenko, D. 2005. Combining reinforcement learning with symbolic planning. In Adaptive Agents and Multi-Agent Systems III. Adaptation and Multi-Agent Learning. Springer. 75-86.

Guez, A.; Mirza, M.; Gregor, K.; Kabra, R.; Racaniere, S.; Weber, T.; Raposo, D.; Santoro, A.; Orseau, L.; Eccles, T.; et al. 2019. An investigation of model-free planning. In ICML, 2464-2473. 
Hengst, B. 2002. Discovering hierarchy in reinforcement learning with HEXQ. In ICML, 243-250.

Hengst, B. 2004. Model approximation for HEXQ hierarchical reinforcement learning. In ECML, 144-155.

Jong, N. K., and Stone, P. 2008. Hierarchical model-based reinforcement learning: R-MAX+MAXQ. In ICML, 432439.

Jonsson, A., and Barto, A. 2005. A causal approach to hierarchical decomposition of factored MDPs. In ICML, 401408.

Kearns, M., and Singh, S. 2002. Near-optimal reinforcement learning in polynomial time. Machine learning 49(23):209-232.

Keller, T., and Eyerich, P. 2012. PROST: probabilistic planning based on UCT. In ICAPS, 119-127.

Kocsis, L., and Szepesvári, C. 2006. Bandit based montecarlo planning. In ECML, 282-293.

Konidaris, G.; Kaelbling, L. P.; and Lozano-Perez, T. 2018. From skills to symbols: Learning symbolic representations for abstract high-level planning. JAIR 61:215-289.

Kulkarni, T. D.; Narasimhan, K.; Saeedi, A.; and Tenenbaum, J. 2016. Hierarchical deep reinforcement learning: Integrating temporal abstraction and intrinsic motivation. In NeurIPS, 3675-3683.

Levy, A.; Platt, R.; Konidaris, G.; and Saenko, K. 2019. Learning multi-level hierarchies with hindsight. In ICLR.

Li, L.; Littman, M. L.; Walsh, T. J.; and Strehl, A. L. 2011. Knows what it knows: a framework for self-aware learning. Machine learning 82(3):399-443.

Li, Z.; Narayan, A.; and Leong, T.-Y. 2017. An efficient approach to model-based hierarchical reinforcement learning. In $A A A I, 3583-3589$.

Li, L. 2012. Sample complexity bounds of exploration. In Reinforcement Learning. Springer. 175-204.

MacGlashan, J.; Babes-Vroman, M. C.; desJardins, M.; Littman, M. L.; Muresan, S.; Squire, S.; Tellex, S.; Arumugam, D.; and Yang, L. 2015. Grounding English commands to reward functions. In RSS

McMahan, H. B.; Likhachev, M.; and Gordon, G. J. 2005. Bounded real-time dynamic programming: RTDP with monotone upper bounds and performance guarantees. In $I C M L$, 569-576.

Mehta, N.; Ray, S.; Tadepalli, P.; and Dietterich, T. 2008. Automatic discovery and transfer of MAXQ hierarchies. In ICML, 648-655.

Mehta, N. 2011. Hierarchical Structure Discovery and Transfer in Sequential Decision Problems. Ph.D. Dissertation, Oregon State University.

Nachum, O.; Gu, S. S.; Lee, H.; and Levine, S. 2018. Dataefficient hierarchical reinforcement learning. In NeurIPS, 3303-3313.

Parr, R., and Russell, S. J. 1998. Reinforcement learning with hierarchies of machines. In NeurIPS, 1043-1049.
Rafati, J., and Noelle, D. C. 2019. Learning representations in model-free hierarchical reinforcement learning. In $A A A I$, 10009-10010.

Segovia-Aguas, J.; Ferrer-Mestres, J.; and Jonsson, A. 2016. Planning with partially specified behaviors. In Artificial Intelligence Research and Development-Proceedings of the 19th International Conference of the Catalan Association for Artificial Intelligence, volume 288, 263-272.

Silver, D., and Ciosek, K. 2012. Compositional planning using optimal option models. In ICML, 1063-1070.

Strehl, A. L.; Li, L.; and Littman, M. L. 2009. Reinforcement learning in finite MDPs: PAC analysis. JMLR 10(Nov):2413-2444.

Sutton, R. S.; Precup, D.; and Singh, S. 1999. Between MDPs and semi-MDPs: A framework for temporal abstraction in reinforcement learning. Artificial intelligence 112(12):181-211.

Szita, I., and Szepesvári, C. 2011. Agnostic KWIK learning and efficient approximate reinforcement learning. In COLT, 739-772.

Taylor, M. E., and Stone, P. 2009. Transfer learning for reinforcement learning domains: A survey. JMLR 10(Jul):16331685 .

Topin, N.; Haltmeyer, N.; Squire, S.; Winder, J.; desJardins, M.; and MacGlashan, J. 2015. Portable option discovery for automated learning transfer in object-oriented Markov decision processes. In IJCAI, 3856-3864.

Vigorito, C. M., and Barto, A. G. 2010. Intrinsically motivated hierarchical skill learning in structured environments. IEEE TAMD 2(2):132-143. 